\title{
The Arab Journal of Interventional Radiology: Launching into the Future
}

Vascular interventional radiology (VIR), driven by its minimally invasive innovative therapies and improved technology, has played an integral role in the practice of medicine over the past few decades. Despite all the challenges and gravity pull that faced the subspecialty worldwide, VIR in the Middle East and Arab World lifted off successfully setting the sky as the limit. The high prevalence of diabetes, peripheral arterial disease, end-stage renal disease, cirrhosis, and hepatocellular carcinoma in the region translates into high demand on interventional radiologists. VIR as a radiology subspecialty was incorporated in fellowship training programs in several countries in the Arab World.

The Pan Arab Interventional Radiology Society (PAIRS) was established in 2006, as the largest and leading VIR professional body. PAIRS has taken several remarkable steps to expand the interventional radiology network in the region and enhance collaboration with other societies worldwide. PAIRS also recognized the need to enhance VIR research in the region given the specific patient population characteristics and economic and cultural factors.

During the $3^{\text {rd }}$ PAIRS 2016 annual meeting in Dubai, the board of directors approved establishing the Arab Journal of Interventional Radiology (AJIR) as the official scientific journal to further promote VIR at the societal and academic levels.

The founding editorial board of AJIR, represented by experts in different aspects of the field, sets its vision to enhance Visibility, Integrity, and promote Research in the Middle East and the Arab World.

Visibility: The journal offers a worldwide open access to its contents and it is free of submission and publication charges to increase accessibility and enhance dissemination of knowledge. AJIR accepts original research, reviews, letters to editor, and case reports. AJIR will be the platform to publish accepted abstracts in the PAIRS annual meetings.

Integrity: The journal follows several measures to ensure high quality of published research and prevent scientific misconduct, including thorough editorial and peer review process and plagiarism checks. The journal strictly adheres to research and publication ethics set by the Committee on Publication Ethics and the International Conference on Harmonization guidelines for Good Clinical Practice.
Research: PAIRS recognizes research and innovation as the most credible "vital sign" of its academic activities and the mainstay of advancing interventional radiology in the Arab World. While clinical practice of VIR had significantly improved during the past decades in the Arab countries, research output by the practicing interventional radiologists had not been at the expected level when compared to the large volume and diversity of procedures being done. Translating clinical practice into organized research projects, and in turn into local practice guidelines, requires extensive efforts and multi-institutional collaboration. PAIRS takes the lead responsibility to promote "research habits" in the field of interventional radiology and increase the visibility of academic institutions by participating in worldwide registries and clinical trials, organizing multi-institutional studies and setting guidelines that fit the practice and patient population in the region.

As we present AJIR to our readers, we are grateful to all authors who contributed their work to this inaugural issue, and we invite interventional radiologists and other practitioners in the region and worldwide to submit their research to future AJIR issues. We are looking forward to provide a distinguished platform and promoting high-quality research in the region.

Mohammad Arabi, Saher Sabri ${ }^{1}$

Department of Medical Imaging, Vascular Interventional Radiology, King Abdulaziz Medical City, Riyadh, KSA, ${ }^{I}$ Department of Radiology, Vascular Interventional Radiology, University of Virginia Health System, VA, USA. E-mail:marabi2004@hotmail.com

This is an open access article distributed under the terms of the Creative Commons Attribution-NonCommercial-ShareAlike 3.0 License, which allows others to remix, tweak, and build upon the work non-commercially, as long as the author is credited and the new creations are licensed under the identical terms.

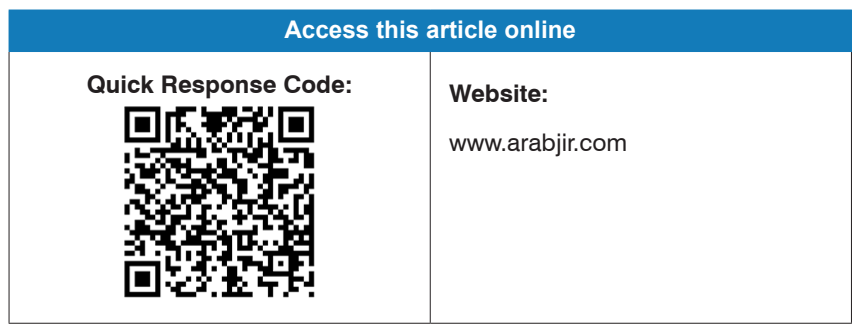

How to cite this article: Arabi M, Sabri S. The Arab Journal of Interventional Radiology: Launching into the Future. Arab J Intervent Radiol 2017;1:1. 